\title{
An Anderson Impurity in a Semiconductor
}

\author{
Clare C. Yu and M. Guerrero ${ }^{\dagger}$ \\ Department of Physics and Astronomy, University of California, Irvine, CA 92717-4575
}

(November 28, 2017)

\begin{abstract}
We study an Anderson impurity in a semiconducting host using the density matrix renormalization group technique. We use the $U=0$ one-dimensional Anderson Hamiltonian at half filling as the semiconducting host since it has a hybridization gap. By varying the hybridization of the host, we can control the size of the semiconducting gap. We consider chains with 25 sites and we place the Anderson impurity (with $U>0$ ) in the middle of the chain. We dope the half-filled system with one hole and we find two regimes: when the hybridization of the impurity is small, the hole density and the spin are localized near the impurity. When the hybridization of the impurity is large, the hole and spin density are spread over the lattice. Additional holes avoid the impurity and are extended throughout the lattice. Away from half-filling, the semiconductor with an impurity is analogous to a double well potential with a very high barrier. We also examine the chemical potential as a function of electron filling, and we find that the impurity introduces midgap states when the impurity hybridization is small.
\end{abstract}

$\dagger$ Current address: T-11, MS B262, Los Alamos National Laboratory, Los Alamos, NM 87545 . 


\section{INTRODUCTION}

It is well known that a magnetic Anderson impurity in a metal is screened by conduction electrons, and a singlet is formed at low temperatures. However, very little attention has been given to the nature of the ground state when a magnetic impurity is in an insulator or a semiconductor. That is the subject of this paper. One might think that a magnetic impurity in an insulator or a semiconductor will not be screened because there is a gap in the density of states. However, the problem is somewhat more complicated than this simple expectation.

Let us briefly review the previous work in this field. Withoff and Fradkint used mean field theory to consider a Kondo impurity in a system where the density of states goes to zero at the Fermi energy with power law behavior, i.e., $\rho(\epsilon) \sim|\epsilon|^{r}$ where $|\epsilon|<D(D$ is the bandwidth) and $r=0,1 / 2,1$, or 2 . In this gapless situation they found that the Kondo impurity became a singlet only if the spin exchange $J>J_{c}$, where the critical coupling $J_{c}$ was a function of the power $r$. Later authors considered a finite energy gap $\Delta$ in the density of states. Takegahara et al. $\mathrm{\text {Q }}$ used Wilson's numerical renormalization group to argue that the symmetric Anderson impurity is always a magnetic multiplet for any finite gap $\Delta$, while the asymmetric Anderson impurity 3 has a critical value of the gap $\Delta_{c}$ such that the impurity has a magnetic ground state if the gap is too big, i.e., if $\Delta>\Delta_{c}$. Ogura and Sasol found no such qualitative difference between the symmetric and asymmetric impurities. They have examined the problem using poor man's scaling, the $1 / \mathrm{N}$ expansion, the noncrossing approximation, and quantum Monte Carlo. They argued that both the symmetric and asymmetric Anderson impurity remain magnetic if the semiconducting gap $\Delta$ is "large enough"; otherwise the impurity is screened and forms a singlet at low temperatures. "Large enough" means that $\Delta \geq \alpha T_{K}$, where $T_{K}$ is the Kondo temperature and the value of $\alpha$ varies

between 0.4 and 2.0, depending on the parameters and the calculational approach. Since there is a gap, it is somewhat artificial to define a Kondo temperature, but Ogura and Saso define it by $T_{K}=D \exp \left(-1 / J \rho_{o}\right)$, where $\rho_{o}$ is the flat density of states of the semiconductor 
outside the gap.

All of these considerations focus on whether or not the impurity is screened and becomes a singlet at low temperatures. This tacitly assumes that the magnetic moment is localized at the impurity site. This is not necessarily true. As we shall see, if there is a large hybridization between the localized orbital and the conduction orbital on the impurity site, the spin and charge are not localized at the impurity site, but are extended throughout the system. The importance of hybridization is well known in semiconductors. After all, it is hybridization that allows ordinary donor and acceptor impurities to contribute carriers to a semiconductor. Within the Hartree-Fock approximation, Haldane and Anderson 5 found that hybridization between the d-orbitals of a transition metal impurity and the valence band electrons of the semiconductor allowed nominally different charge states to exist as states in the gap.

In this paper we place an Anderson impurity in the middle of a one-dimensional semiconductor. We use the $U=0$ one-dimensional Anderson Hamiltonian at half filling as the semiconducting host, since it has a hybridization gap. By varying the hybridization $V$, we can control the size of the semiconducting gap. We consider chains with an odd number of sites, and we place the substitutional Anderson impurity in the middle of the chain. The impurity has a positive Coulomb repulsion $U_{0}>0$ and a positive hybridization $V_{0}$. In section II we present the Hamiltonian, which we study using the density matrix renormalization group approach. The advantage of this technique is that it is done in real space so that we can evaluate how correlation functions vary with distance as well as examine the spin and charge densities as a function of position.

In section IIIA we discuss the half-filled case; we find that the ground state always has total spin $S=0$. We also study the spin-spin correlation functions to see if the impurity spin persists spatially. We find that the spin correlations decay exponentially with distance due to the presence of the gap. We compare these results to the metallic case in which we place the Anderson impurity in a free electron host with the same number of sites as in the semiconducting case. In the metallic case we find that the spin correlations decay more slowly with distance. We would also like to plot the spin as a function of position. 
However, the half-filled case has a singlet ground state with $S_{i}=0$ on each site. So in section IIIB, we consider the doped case in which we add a hole to the system; this makes the total spin $S=1 / 2$. In the doped semiconducting case we find two different regimes. When the hybridization of the impurity site is small, the spin and charge of the hole are localized near the impurity. When the hybridization is large, the spin and charge of the hole are delocalized and reside in the host away from the impurity; a singlet is formed on the impurity site. Thus, in the semiconducting case, the spin and the hole go together and have similar spatial distributions. In contrast, in the doped metallic case, the charge density of the hole is delocalized for all values of the impurity hybridization $V_{0}$. However, the spin density of the hole is localized near the impurity for small values of the hybridization due to finite size effects. For large values of $V_{0}$, finite size effects no longer dominate; a singlet forms on the impurity site, and the hole and spin densities are delocalized.

When more than one hole is added to the semiconductor, the additional holes avoid the impurity and spread throughout the lattice. We show that there is an analogy between the barrier in a double well potential and the impurity in the semiconductor doped away from half-filling. Finally in section IIIc, we discuss how the chemical potential varies with filling. For small $V_{0}$, we find states lying in the middle of the gap. As $V_{0}$ increases, these states move toward the edges of the gap.

\section{THE 1D ANDERSON HAMILTONIAN WITH AN IMPURITY}

The standard one-dimensional periodic Anderson lattice has spin-1/2 conduction electrons that hop from site to site. Each site has a localized f-orbital with a Coulomb repulsion $U(i)$ and a hybridization $V(i)$ between the conduction orbital and the f-orbital. The Hamiltonian is given by:

$$
H=-t \sum_{i \sigma}\left(c_{i \sigma}^{\dagger} c_{i+1 \sigma}+c_{i+1 \sigma}^{\dagger} c_{i \sigma}\right)+\sum_{i \sigma} \varepsilon_{f}(i) n_{i \sigma}^{f}+\sum_{i} U(i) n_{i \uparrow}^{f} n_{i \downarrow}^{f}+\sum_{i \sigma} V(i)\left(c_{i \sigma}^{\dagger} f_{i \sigma}+f_{i \sigma}^{\dagger} c_{i \sigma}\right)
$$

where $c_{i \sigma}^{\dagger}$ and $c_{i \sigma}$ create and annihilate conduction electrons with spin $\sigma$ at lattice site $i$, and $f_{i \sigma}^{\dagger}$ and $f_{i \sigma}$ create and annihilate local $f$-electrons. Here $t$ is the hopping matrix element for 
conduction electrons between neighboring sites, $\varepsilon_{f}(i)$ is the energy of the localized $f$-orbital at site $i, U(i)$ is the on-site Coulomb repulsion of the $f$-electrons and $V(i)$ is the on-site hybridization matrix element between electrons in the $f$-orbitals and the conduction band. For simplicity, we neglect orbital degeneracy. We denote the number of electrons by $N_{e l}$, and $L$ is the number of sites in the lattice. $t, U(i), V(i)$, and $\varepsilon_{f}(i)$ are taken to be real numbers.

In order to find the semiconducting gap $\Delta$, we note that the uniform periodic Anderson Hamiltonian with $U(i)=0$ and $V(i)=V$ independent of $i$ can be diagonalized exactly in $k$ space. We obtain two hybridized bands with energies $\lambda_{k}^{ \pm}$:

$$
\lambda_{k}^{ \pm}=\frac{1}{2}\left[\left(\varepsilon_{f}-2 t \cos (k a)\right) \pm \sqrt{\left(\varepsilon_{f}+2 t \cos (k a)\right)^{2}+4 V^{2}}\right]
$$

where $a$ is the lattice constant. When there are two electrons per unit cell, the lower band is full while the upper one is empty. Thus the system is insulating when $N_{e l}=2 L$. The size of the band gap is given by

$$
\Delta=\lambda_{k=0}^{+}-\lambda_{k=\pi / a}^{-}=2 \sqrt{t^{2}+V^{2}}-2 t
$$

In this paper we consider chains with an odd number of sites and we place the impurity in the center of the chain. We denote the site at the middle by $i=0$. (For example, for a 25 site chain, $i$ runs from -12 to +12 .) For $i \neq 0$, we set $U(i)=0$ and $V(i)=V$. At half filling $\left(N_{e l}=2 L\right)$, these sites represent the semiconducting host since there is a hybridization gap between the conduction band and the flat $\mathrm{f}$-band. We set $t=1$; this sets the energy scale. By varying the value of $V$ we can control the size of the gap. The impurity site at $i=0$ has $U(0)=U_{0}, V(0)=V_{0}$, and $\varepsilon_{f}(0)=\varepsilon_{f 0}$.

We primarily consider the symmetric case, for which $\varepsilon_{f}(i)=-U(i) / 2$. With this choice, the half-filled case has particle-hole symmetry and there is an $\mathrm{SU}(2)$ charge pseudospin symmetry 0 The components of the pseudospin operator $\vec{I}$ are given by:

$$
I_{z}=\frac{1}{2} \sum_{i}\left(c_{i \uparrow}^{\dagger} c_{i \uparrow}+c_{i \downarrow}^{\dagger} c_{i \downarrow}+f_{i \uparrow}^{\dagger} f_{i \uparrow}+f_{i \downarrow}^{\dagger} f_{i \downarrow}-2\right)
$$




$$
\begin{aligned}
& I_{+}=\sum_{i}(-1)^{i}\left(c_{i \uparrow}^{\dagger} c_{i \downarrow}^{\dagger}-f_{i \uparrow}^{\dagger} f_{i \downarrow}^{\dagger}\right) \\
& I_{-}=\sum_{i}(-1)^{i}\left(c_{i \downarrow} c_{i \uparrow}-f_{i \downarrow} f_{i \uparrow}\right)
\end{aligned}
$$

The $\mathrm{z}-$-component of the pseudospin is equal to $\left(N_{e l} / 2\right)-L$. Note that half-filling corresponds to $I_{z}=0$. An $I_{z}=1$ state can be achieved by adding two electrons. All the energy eigenstates of the symmetric Anderson model have a definite value of S and I. At half filling the ground state is a singlet both in spin and pseudospin space $(S=0, I=0)$, even when an impurity is present.

Since Takegahara et al 目目 have emphasized the difference between a symmetric and an asymmetric Anderson impurity, we also consider an asymmetric Anderson impurity in a symmetric $U=0$ Anderson lattice. We find no qualitative difference between the symmetric case and the asymmetric case.

We compare our results for the semiconductor to the metallic case in which we place the Anderson impurity in the center of a one-dimensional free electron host with the same number of sites as in the semiconducting case. The Hamiltonian for the metallic case is given by

$$
H=H_{o}+H_{i m p}
$$

where the free electron tight--binding Hamiltonian $H_{o}$ is given by

$$
H_{o}=-t \sum_{i \sigma}\left(c_{i \sigma}^{\dagger} c_{i+1 \sigma}+c_{i+1 \sigma}^{\dagger} c_{i \sigma}\right)
$$

and the impurity Hamiltonian $H_{i m p}$ is given by

$$
H_{i m p}=\sum_{\sigma} \varepsilon_{f}(0) n_{0 \sigma}^{f}+U(0) n_{0 \uparrow}^{f} n_{0 \downarrow}^{f}+\sum_{\sigma} V(0)\left(c_{0 \sigma}^{\dagger} f_{0 \sigma}+f_{0 \sigma}^{\dagger} c_{0 \sigma}\right)
$$

For future reference, it is convenient to define the effective spin exchange coupling $J_{\text {eff }}$. This comes from considering a single Anderson impurity in a metal. When the hybridization term is small $\left(\pi \rho\left(\varepsilon_{f}+U\right) V^{2} /\left(\epsilon_{f}+U\right) \ll 1\right.$ and $\left.\pi \rho\left(\varepsilon_{f}\right) V^{2} / \epsilon_{f} \ll 1\right)$, the single impurity Anderson Hamiltonian can be mapped into the Kondo Hamiltonian 


$$
H_{K}=J_{e f f} \vec{S}^{f} \cdot \vec{s}_{0}^{c}
$$

where $\vec{s}_{0}^{t}$ is the spin density of the conduction electrons at the impurity site and $J_{\text {eff }}$ is given by the Schrieffer-Wolff transformation:8

$$
J_{e f f}=-\frac{2|V|^{2} U}{\epsilon_{f}\left(\epsilon_{f}+U\right)}
$$

Note that for the symmetric case where $\epsilon_{f}=-U / 2, J_{\text {eff }}=8 \mathrm{~V}^{2} / U$.

We use the density matrix renormalization group (DMRG) methodf to calculate the ground state as well as to determine the spin-spin correlation functions, and the spin and charge densities in the ground state. The DMRG appoach is a real space technique which has proven to be remarkably accurate for one dimensional systems such as the Kondo and Anderson lattices. 0.0 We use the finite system method with open boundary conditions in which there is no hopping past the ends of the chain. We study lattices with 25 sites, keeping up to 120 states with typical truncation errors of order $10^{-7}$ for the semiconductor. For the metal we keep up to 130 states with typical truncation errors of order $10^{-6}$.

\section{RESULTS}

\section{A. Half-Filled Case}

In this section we consider the half-filled case. In particular we want to see how far the impurity spin persists by studying the spin-spin correlation functions. We compare the gapped semiconducting case with the gapless metallic case. We find that the spin correlations fall off much faster in the presence of a gap.

To model the semiconductor, we use Anderson lattice chains with 25 sites and $N_{e l}=$ $2 L=50$ (2 electrons per site or one electron per orbital). The Hamiltonian is given by eq. (1) with $U=\epsilon_{f}=0$ for $i \neq 0$, and $U_{0}>0$ and $\epsilon_{f 0}=-U_{0} / 2$ for the symmetric impurity site with $i=0$. We set $t=1$. For $V=1$, the semiconducting gap $\Delta \sim 0.83$ for a 25 site chain with open boundary conditions.11 We initially set $U_{0}=8$ and $V_{0}=1$ at the impurity site. 
We model the half-filled metal with the tight-binding Hamiltonian of eqs. (5,6,7). In this case $N_{e l}=26$. We set $t=1$ and place the impurity at the center at site $i=0$ with the same values of $U_{0}$ and $V_{0}$ that we used for the semiconductor.

For both the metallic and semiconducting cases, we find that the ground state has $I=0$ and $S=0$. We show the spin-spin correlation functions for both cases in Fig. 11. We plot the correlations $<S_{z}^{c}(R) S_{z}^{f}(0)>$ between the z-component of the f-spin at the impurity site and the $\mathrm{z}$-component of the spin of the conduction electrons in the lattice versus the distance $R$ from the impurity. Fig. [1a is a linear plot. The metallic case clearly shows Friedel oscillations. The bias of the data for the metal toward negative values of the correlations is a finite size effect; we show in the appendix that the leading term in perturbation theory for $<S_{z}^{c}(R) S_{z}^{f}(0)>$ goes as $-1 / L$. Fig. 四 is a linear-log plot of the absolute value of the same correlations; we have removed the oscillations by plotting every other point. Here we see that in the metallic case the correlations between the impurity f-spin and the conduction spins, which are responsible for the compensation of the magnetic moment, decay very slowly. In contrast, in the semiconducting case the decay is much faster due to the presence of a gap in the excitation spectrum. If assume that the correlations fall off exponentially and fit the plots in Fig. 1 $\mathrm{b}$ to the form $\exp (-R / \xi)$, then the correlation length $\xi / a \sim 1.9$ for the metal and $\xi / a \sim 0.49$ for the semiconductor.

We attribute the fact that the spin correlations decay much faster in the semiconductor than in the metal due to the presence of semiconducting gap. To check this, we can change the size of the gap in the semiconductor by varying $V$. This should change the spin-spin correlation length. This is confirmed in Fig. 1 where we plot the spin-spin correlation functions for the semiconducting case for 2 different values of $V$ in a linear-log plot with $U_{0}=8$. For $V=0.4, \Delta \sim 0.16$, and $\xi / a \sim 0.95 ;$ for $V=1, \Delta \sim 0.83$, and $\xi / a \sim 0.49 .11$ Thus we see that as $V$ and hence the gap $\Delta$ increase, the correlation length decreases as expected.

We varied the hybridization $V_{0}$ at the impurity site to study the effect on the spinspin correlation functions at half-filling for both the metallic and the semiconducting cases. 
In the semiconducting case, we find that changing $V_{0}$ from 0.5 to 10 , while keeping the semiconducting gap constant, does not change the qualitative behavior of the correlation functions. Similarly, in the metallic case we find that changing $V_{0}$ from 0.1 to 10 does not change the qualitative behavior of the correlation functions.

We also examined an asymmetric Anderson impurity in a semiconductor at half-filling with $L=25, N_{e l}=50, t=1, V=1, U=0, U_{0}=16$, and $\varepsilon_{f 0}=-0.5$. We found no qualitative difference in the spin-spin correlation functions as $V_{0}$ varied between 0.1 and 10 . In fact, the the behavior of the correlation functions was very similar to that found in the symmetric case.

\section{B. Doped Case}

If we plot the spin and charge density as a function of position in the half-filled case, we find that the spin is zero and the charge density is 2 at every site. In order to obtain more interesting positional information, we dope our system of 25 sites by adding a hole. We put $N_{e l}=49$ in the semiconducting case and $N_{e l}=25$ in the metallic case. The total spin in the ground state is $1 / 2$ since there is an odd number of electrons. This corresponds to a quasiparticle excitation of the half-filled system. Again, we set $t=1, V=1$, and $U=0$ in the host. By fixing $V$, we set the value of the gap $\Delta=0.83$ in the semiconducting

case. We initially consider a symmetric Anderson impurity with $U_{0}=8$ and we vary $V_{0}$. One can think of changing $V_{0}$ as corresponding to changing the effective Kondo coupling $J_{\text {eff }}=8 V_{0}^{2} / U_{0}$, although this picture is only valid for small $V_{0}$.

We study the hole density and the spin density versus site. (Here the hole density refers to the number of holes per site measured relative to the half-filled case.) Naively, we can think of two possibilities: the hole can be localized in the impurity site or it can be delocalized and spread out in the rest of the chain. The results for the semiconducting case appear in Fig. 3. We can clearly identify two regimes in the semiconductor:

i) Large $V_{0}$ : the hole and the spin density are delocalized and reside in the host. On 
the impurity site the hole and spin density are zero, which means that the impurity has an f-electron and a conduction electron combined in a singlet state.

ii) Small $V_{0}$ : the spin density is localized at the impurity site while the hole density is localized on the impurity site and its nearest neighbors.

To understand what determines whether or not the hole is localized, we must compare the energy of adding a delocalized hole to the host versus the energy of adding a hole to the impurity. Removing an electron from the semiconductor costs roughly half the gap $(\Delta / 2)$. To estimate the energy of putting a hole on the impurity, we consider the Hamiltonian of an isolated single site Anderson impurity:

$$
H_{0}=U_{0} n_{\uparrow}^{f} n_{\downarrow}^{f}-\frac{U_{0}}{2}\left(n_{\uparrow}^{f}+n_{\downarrow}^{f}\right)+V_{0} \sum_{\sigma}\left(c_{\sigma}^{\dagger} f_{\sigma}+f_{\sigma}^{\dagger} c_{\sigma}\right)
$$

Since this is a symmetric impurity, we can classify the states by their value of $S$ and $I$. We find

- $2(S=0, I=0) \quad$ states with $\quad E_{ \pm}^{00}=-\frac{U_{0}}{4} \pm \frac{1}{2} \sqrt{\frac{U_{0}^{2}}{4}+16 V_{0}^{2}}$

- $2(S=1 / 2, I=1 / 2) \quad$ states with $\quad E_{ \pm}^{\frac{1}{2} \frac{1}{2}}=-\frac{U_{0}}{4} \pm \frac{1}{2} \sqrt{\frac{U_{0}^{2}}{4}+4 V_{0}^{2}}$

- $1(S=1, I=0) \quad$ state with $\quad E^{10}=-\frac{U_{0}}{2}$

- $1(S=0, I=1) \quad$ state with $\quad E^{01}=0$

Here the first superscript of $E$ indicates the value of $S$ and the second one indicates the value of $I$ in that state. For a single impurity, the $I=0$ states have two electrons, while the $I=1 / 2$ states can have 3 electrons $\left(I_{z}=+1 / 2\right)$ or one electron $\left(I_{z}=-1 / 2\right)$.

The lowest energy state is the lowest state with $S=0, I=0$ for any choice of parameters. In general we find

$$
E_{-}^{00}<E_{-}^{\frac{1}{2} \frac{1}{2}}<E^{10}<E^{01}<E_{+}^{\frac{1}{2} \frac{1}{2}}<E_{+}^{00}
$$

The difference in energy $\Delta E$ between the two lowest states is

$$
\Delta E \equiv E_{-}^{\frac{1}{2} \frac{1}{2}}-E_{-}^{00}
$$


For large $V_{0}\left(16 V_{0}^{2} \gg U_{0}^{2} / 4\right)$ one gets $\Delta E \sim V_{0}$ and for small $V_{0}, \Delta E \sim 6 V_{0}^{2} / U$. This difference $\Delta E$ represents the energy cost to put the hole at the impurity site. If, on the other hand, the hole goes to the host, the energy cost is roughly equal to half the gap $(\Delta / 2)$. Therefore, when $\Delta E<\Delta / 2$, the hole should go to the impurity site, meaning that the impurity should be in the $S=1 / 2, I=1 / 2$ state with $S_{z}=1 / 2$ and $I_{z}=-1 / 2$. According to this criteria, the crossover should occur when $\Delta E=\Delta / 2$. For the values of the parameters that we use, this crossover corresponds to $V_{0} \sim 1.25$. For $V_{0}$ less than 1.25, the hole and the spin density should be localized at the impurity site because the $S=1 / 2, I=1 / 2$ state is more favorable, but when $V_{0}$ is greater than 1.25 , the impurity should be in the $S=0, I=0$ state and the hole and the spin density should be spread out over the lattice. This is consistent with the numerical results, since for $V_{0}=1$ the hole is localized while for $V_{0}=2$ it is spread out over the lattice. In the crossover region $\left(1<V_{0}<2\right)$ the values of the hole and spin densities on the impurity site are intermediate between those found for $V_{0}=1$ and $V_{0}=2$. However, this gradual crossover may be a finite size effect since we have only looked at lattices up to 25 sites long.

Fig. 3 shows that for small $V_{0}\left(V_{0}<1.25\right)$, the hole density is localized on the impurity as well as its nearest neighbor sites. This can be understood as follows: the hole density likes to be localized at the impurity according to the criteria explained above. However, electrons on neighboring sites optimize their kinetic energy by hopping into the hole on the impurity site. Thus the hole spreads to the two nearest neighbor sites of the impurity. This is confirmed in Fig. 1 which shows of the kinetic energy of the bonds between sites as a function of position. Notice that the bonds connecting the impurity site have the largest magnitude of the kinetic energy for the smallest values of $V_{0}$. We have looked at the conduction and $\mathrm{f}-$ electron density on the sites neighboring the impurity. We find that when the hole resides on these sites, it is primarily in the f-orbital where the energy cost is zero because $\varepsilon_{f}(i \neq 0)=-U / 2=0$ on these sites. The electrons on these sites are in the conduction orbitals where they can take advantage of the kinetic energy.

Let us discuss what dictates where the spin of the hole resides. When the impurity 
hybridization $V_{0}$ is large, a singlet forms between the conduction spin and the f-spin on the impurity site. Thus the impurity has no net spin, and the spin of the hole resides in the host. Will it reside primarily in the f-orbitals or in the conduction orbitals? To answer this, we note that if the hybridization $V$ of the host is not too large, then optimizing the kinetic energy of the conduction electrons dominates over optimizing the hybridization energy of the host. In order to allow both up and down spin conduction electrons to hop freely from site to site, the average spin of the conduction electrons on each site is zero. Thus the spin of the hole must be spread primarily over the f-orbitals of the host lattice.

On the other hand, when the impurity hybridization $V_{0}$ is small, the hybridization on the host sites has priority. This favors the formation of singlets on the host sites. As a result, the spin of the hole will be localized primarily on the impurity site. In order to minimize the kinetic energy of the conduction electrons, the spin will primarily reside in the $\mathrm{f}-$ orbital of the impurity. This occurs at the expense of the hybridization energy of the impurity, but that is permissible since this is the smallest energy in the problem. The arguments of the last two paragraphs indicate that the spin of the hole will be primarily in the f-orbitals for the range of parameters that we studied. This is shown in Fig. 5 .

We now consider the metallic case. It is easy to compare the energy of adding the hole to the host versus the finite energy $\Delta E$ of localizing the hole on the impurity. For a metallic host, the chemical potential is zero at half-filling, and there is no energy cost in adding a delocalized hole to the metal. Thus, one expects that the hole will always be spread out and extended throughout the metal. In Fig. [6 we show the numerical results. We see that the hole density behaves as expected: it is spread out over the lattice for every set of parameters that we examined. For large $V_{0}$, the large on-site hybridization favors a singlet state at the impurity and the spin density is spread out over the lattice. In this case the spin is in the conduction spins because there are no f-orbitals in the metallic host. However, for small values of $V_{0}$, the spin density becomes localized at the impurity. We attribute this to finite size effects since we expect a singlet at the impurity site in an infinite metallic lattice. We can understand how finite size effects affect the behavior of the spin density in the following 
way. If the size of the lattice is such that the spacing between discrete energy levels of the metallic host becomes comparable to or larger than $J_{\text {eff }}$, then the exchange interaction is too weak to mix the noninteracting conduction energy levels enough to form a singlet with the f-spin. In this case, there will be a magnetic moment on the impurity site. We can check this explanation by comparing $J_{\text {eff }}$ with the energy level spacing. For a 25 site metallic lattice with open boundary conditions, the typical energy level spacing is 0.24 . We can compare this with $V_{0}=0.1$ which has $J_{\text {eff }}=0.01$, and with $V_{0}=1$ which has $J_{e f f}=1$. As we can see in Fig. Gb these two cases have a local magnetic moment. The $V_{0}=2$ case is borderline and has a small magnetic moment at the impurity site. The influence of finite size effects can be seen by putting a symmetric Anderson impurity with $V_{0}=2$ and $U=8$ in the middle of a 7 site lattice. We find that the spin density on the impurity site is roughly twice that found for a 25 site lattice (see Fig. 6b). Finally we note that finite size effects do not affect our results for a semiconductor because the semiconducting gap is much larger than the energy level spacing. For example, a 25 site symmetric Anderson lattice with open boundary conditions with $U=0$ and $V=1$ has a typical energy level spacing of 0.01 which is much smaller than the semiconducting gap of $\Delta=0.83$. Similarly if $V$ is changed to 0.4 , the energy level spacing is still approximately 0.01 which is much smaller than $\Delta=0.15$.

We also examined the asymmetric Anderson impurity in a semiconductor doped with either one hole or one particle with $L=25, N_{e l}=49, t=1, V=1, U_{0}=16, \varepsilon_{f 0}=-0.5$. The behavior of the spin and charge densities at small $V_{0}\left(V_{0}=0.1\right)$ and at large $V_{0}\left(V_{0}=10\right)$ is very similar to that found for the symmetric Anderson impurity.

We will devote the rest of this section to discussing the fact that the impurity provides a large potential barrier and effectively divides the lattice in two as the system is doped away from half-filling. As a result, we can think of the semiconductor as a symmetric double well potential. There are several examples of where this occurs. For example, consider what happens when we add two holes to a half-filled semiconductor with a symmetric Anderson impurity. As before, we set $t=1, V=1$, and $U=0$ in the host. We place the Anderson impurity in the center of a 25 site lattice with $U_{0}=8$, and we vary $V_{0}$. Adding two holes 
corresponds to $N_{e l}=48$. For small $V_{0}$, the ground state consists of two states which are degenerate within the accuracy of our calculation.12 In one state the system is a singlet and in the other it is a triplet. This near degeneracy is not the result of finite size effects or boundary conditions since we find this degeneracy for smaller lattice sizes as well as for the case of periodic boundary conditions. By examining the hole density versus site as shown in Fig. 7, we find that one hole is localized on the impurity site and its two nearest neighbors, while the other hole is spread over the lattice. We cannot put two holes on the impurity because that would involve removing the f-electron from the impurity which would cost an energy of $U_{0} / 2$. As a result, the additional hole avoids the impurity and its two nearest neighbors, and spreads over the host. It resides primarily in the f-orbitals where the energy $\varepsilon_{f}(i)=-U / 2=0$.

The impurity site with the hole localized in its vicinity acts like an nearly infinite potential barrier to the second hole and effectively divides the lattice in two. Thus, the energy associated with adding the second hole should be equal to that of adding a hole to a $22-$ site semiconductor $(t=1, V=1$, and $U=0)$ with no impurity but with a break $(t=0)$ in the middle. We can think of this semiconductor as a symmetric double well potential with a nearly infinite barrier. Each potential well corresponds to an 11-site semiconductor. Putting a hole on the right side or on the left side or taking a linear combination of these two cases results in states whose energies are nearly degenerate. (This explains the degeneracy of the ground state.13) Within the limits of our accuracy, we find that the energy to put one hole in an 11-site half-filled semiconductor with no impurity is indeed equal to the energy to add a second hole to a 25-site semiconductor with an impurity in the middle. Our double well scenario is further confirmed by the fact that the energy associated with adding the second hole is the same for $V_{0}=0.1$ and $V_{0}=1$ within our accuracy. This is consistent with having a very high barrier for both cases.

On the other hand, for large $V_{0}$, we find that both holes go into the host lattice and a singlet forms between the f-electron and the conduction electron on the impurity site. This singlet acts like a potential barrier, but since having two holes on one side of the 
barrier versus having one hole on each side are not degenerate states, the ground state is nondegenerate. In fact, the ground state of the whole system is a singlet. However, if we keep $V_{0}$ large but have one hole rather than two holes, the singlet on the impurity acts like a very high barrier which divides the wavefunction for the hole into two pieces (see Fig. (3). Since having the hole on one side of the impurity versus the other are nearly degenerate configurations, the ground state is nearly degenerate and both states have spin-1/2.

It is easy to generalize these trends to cases where more than one hole is doped into a half-filled system. For small $V_{0}$, the first hole resides in the vicinity of the impurity, and the additional holes avoid the impurity and are extended throughout the lattice. For large $V_{0}$, a singlet forms on the impurity site; the holes avoid the impurity and are extended throughout the lattice. For both large and small $V_{0}$, the ground state is nearly degenerate when the number of extended holes is odd. For example, when there are four holes and $V_{0}$ is small, one hole resides in the vicinity of the impurity; the remaining 3 holes are spread over the rest of the lattice, and the ground state is nearly degenerate.

\section{Chemical Potential Versus Filling}

In this section we study how the chemical potential varies with electron filling. As in the previous sections, we consider a symmetric Anderson lattice with $t=1, U=8, V=1$, and $L=25$ with the impurity site in the middle of the lattice. We define the chemical potential by

$$
\mu(N)=E(N)-E(N-1)
$$

where $E(N)$ is the ground state energy with $N$ electrons. Our results are shown in Fig. 8. When the impurity is absent, there is a jump in the chemical potential that is centered about half-filling $(N=50)$. This is the quasiparticle gap. From Fig. 8, we see that there are states in the gap for small $V_{0}$. The chemical potential of these midgap states corresponds to the energy of adding a particle or a hole to the half-filled system. These midgap states 
move to the edges of the gap as $V_{0}$ increases. Indeed, they appear to merge with the gap edges for $V_{0} \geq 2$. The fact that the impurity does not seem to affect the chemical potential for large values of $V_{0}$ is consistent with the delocalization of the hole density and its spin which we saw in the last section. The presence of midgap states for small values of $V_{0}$ is consistent with the localization of the hole and its spin. To see this, suppose that $V_{0} \ll V$. Then the $\mathrm{f}-$ orbital on the impurity decouples from the rest of the lattice. In addition the large hybridization $V$ favors having one conduction electron and one f-electron on each of the host sites. As a result, when we put 0,1 , or 2 conduction electrons on the impurity site, the associated particles or holes will be localized in the vicinity of the impurity, and the energies of these states will be nearly degenerate. This means that the chemical potential corresponding to adding a particle or a hole to a half-filled system is close to zero. This is indeed what we see for $V_{0}=0.1$.

\section{CONCLUSIONS}

In this paper we have studied an Anderson impurity in a one-dimensional semiconductor. Although we primarily concentrated on a symmetric Anderson impurity, we found no qualitative difference in behavior between an asymmetric impurity in the mixed valence regime and a symmetric impurity in the Kondo regime. In the undoped half-filled case we found spin-spin correlation functions that decay rapidly with distance due to the gap in the excitation spectrum. This is in contrast with the metallic case in which a much slower decay is seen.

Because DMRG is a real space technique, we were able to go beyond the question of whether or not the magnetic impurity is screened in the presence of a gap in the density of states. In the case of doping with an $S=1 / 2$ hole, we found that a large on-site hybridization $V_{0}$ led to the formation of a singlet on the impurity site and the delocalization

of the spin and charge density throughout the lattice. For small $V_{0}$, the magnetic moment of the hole was localized on the impurity site, and the charge density was concentrated on the 
impurity and its nearest neighbors. The criteria for defining these two regimes was whether it costs more energy to put the hole on the impurity site or to spread it throughout the lattice. This is different from the criteria used by Ogura and Saso ${ }^{0}$ who found that the impurity remained a magnetic multiplet if the semiconducting gap $\Delta$ was greater than some fraction of the Kondo temperature $T_{K}$. It is somewhat artificial to define a Kondo temperature since there is a gap at the Fermi energy, but let us define it by $T_{K}=D \exp \left(-1 / J_{\text {eff }} \rho_{o}\right)$, where $\rho_{o}=2 / \pi^{2} t$ is the density of states at the Fermi energy for free electrons with open boundary conditions, and $D=4 t$ is an estimate of the conduction electron bandwidth. Then we can compare our results with those of Ogura and Saso. We find that the charge and spin density of the hole are localized for $\Delta>T_{K}$, and are extended for $\Delta<T_{K}$. This agrees qualitatively with Ogura and Saso.

We compared our semiconducting results with those of a metal. When we put a hole into the half-filled metal, we find that a singlet forms if $V_{0}$ is large. For $V_{0}$ small, the magnetic moment of the hole is localized on the f-orbital of the impurity due to finite size effects. The charge density of the hole is extended for all values of $V_{0}$ since it always costs less energy to put the hole in an extended wavefunction than to localize it in the vicinity the impurity.

We found that the impurity in a semiconductor doped away from half-filling acts like a barrier in a symmetric double well potential. When $V_{0}$ is large, a singlet forms on the impurity site. This singlet acts like a barrier that divides the lattice in two. The holes in the system avoid the impurity and spread over the rest of the lattice. When $V_{0}$ is small, the first hole goes onto the impurity which acts like a barrier and divides the lattice for the rest of the holes. These additional holes spread over the two halves of the lattice. When the number of delocalized holes is odd, the ground state is nearly degenerate for both large and small values of $V_{0}$.

Finally, we studied the chemical potential as a function of electron filling. We found that midgap states appear for small values of $V_{0}$ and correspond to localization of a hole or particle on the impurity site. As $V_{0}$ increases, these midgap states move towards the edges of the gap which is associated with the delocalization of the hole. 
It may be possible to look for some of the effects we have described in dilute magnetic

semiconductorst ${ }^{\text {t }}$. For example, NMR could be used to determine if the spin-spin correlation length decreases as the semiconducting gap increases. 1 . neglected certain features of those materials such as large g-factors and interactions between impurities. We have also neglected long range Coulomb interactions and the associated screening effects which, for example, come into play between an acceptor ion and the hole it contributes to the valence band. This is a subject for future study.

\section{ACKNOWLEDGMENTS}

This work was initiated by a stimulating discussion with Z. Fisk. We thank S. A. Trugman and H. M. Carruzzo for helpful discussions, and T. Saso for bringing ref. 4 to our attention. This work was supported in part by ONR grant no. N000014-91-J-1502, the Center for Materials Science at Los Alamos National Laboratory, by funds provided by the University of California for the conduct of discretionary research by Los Alamos National Laboratory, and an allocation of computer time from the University of California, Irvine.

\section{APPENDIX}

In this appendix we show that to zeroth order in perturbation theory in a periodic system, the spin-spin correlation function $<0\left|S_{z}^{c}(R) S_{z}^{f}(0)\right| 0>=-1 / 4 L$ in a half-filled one dimensional metal with an odd number of sites and an Anderson impurity at the center. (Thus there is an even number of electrons.) $\mid 0>$ is the ground state of the unperturbed Hamiltonian. To construct the ground state, we note that there is one electron in the $\mathrm{f}_{-}$ orbital of the impurity, and one conduction electron on each site. Since there are an odd number of sites, there are an odd number of conduction spins. If we think of filling the states in the conduction band with conduction electrons, one of the states has an unpaired spin. In the ground state the unpaired conduction spin can form a singlet or a triplet with the f-spin. These two states are degenerate since there are no interactions to zeroth order. Since 
we know that the ground state has $S=0$ in the presence of interactions, we will choose the singlet as the ground state, though we would get the same result if we chose the triplet as the ground state. Thus, we can write:

$$
\mid 0>=\frac{1}{\sqrt{2}}\left[\left|\uparrow_{f} \downarrow_{c}>-\right| \downarrow_{f} \uparrow_{c}>\right]
$$

where $\left|\uparrow_{f} \downarrow_{c}\right\rangle$ denotes an up f-spin and a down conduction spin.

The operator for the $\mathrm{z}^{-}$-component of the conduction spin on a site $R$ is

$$
S_{z}^{c}(R)=\frac{1}{2 L} \sum_{k_{1}, k_{2}} e^{-i\left(k_{1}-k_{2}\right) R}\left(c_{k_{1} \uparrow}^{\dagger} c_{k_{2} \uparrow}-c_{k_{1} \downarrow}^{\dagger} c_{k_{2} \downarrow}\right)
$$

$k$ is a good quantum number because the system has periodic boundary conditions. To zeroth order, the only contribution to $<0\left|S_{z}^{c}(R) S_{z}^{f}(0)\right| 0>$ comes from the $k_{1}=k_{2}=k_{F}$ term of the sum. One can show that the other terms in the sum cancel out. Thus, to lowest order,

$$
<0\left|S_{z}^{c}(R) S_{z}^{f}(0)\right| 0>=-\frac{1}{4 L}
$$

Even though we have derived eq. (16) for a periodic lattice, we expect a similar relation to hold for a chain with open boundary conditions, i.e., we expect $<0\left|S_{z}^{c}(R) S_{z}^{f}(0)\right| 0>=-b / L$ to lowest order, where the constant $b$ is of order unity. 


\section{REFERENCES}

${ }^{1}$ D. Withoff and E. Fradkin, Phys. Rev. Lett. 64, 1835 (1990).

${ }^{2}$ K. Takegahara, Y. Shimuzu, and O. Sakai, J. Phys. Soc. Jpn. 61, 3443 (1992).

${ }^{3}$ K. Takegahara, Y. Shimuzu, N. Goto, and O. Sakai, Physica B 186-188, 381 (1993).

${ }^{4}$ J. Ogura and T. Saso, J. Phys. Soc. Jpn. 62, 4364 (1993).

${ }^{5}$ F. D. M. Haldane and P. W. Anderson, Phys. Rev. B 13, 2553 (1976).

6 T. Nishino and K. Ueda, Phys. Rev. B 47, 12451 (1993).

${ }^{7}$ S. R. White, Phys. Rev. Lett. 68, 3487 (1992); Phys. Rev. B 48,10345 (1993); R.M. Noack, S.R. White and D.J. Scalapino, in Computer Simulations in Condensed Matter Physics VII, Eds. D.P. Landau, K.K. Mon, and H.B. Schüttler (Springer Verlag, Heidelberg, Berlin, 1994), p. 85-98.

${ }^{8}$ J. R. Schrieffer and P. A. Wolff, Phys. Rev. 149, 491 (1966).

${ }^{9}$ M. Guerrero and C. C. Yu, Phys. Rev. B 51, 10301 (1995).

${ }^{10}$ M. Guerrero and R. M. Noack, to be published in Phys. Rev. B.

${ }^{11}$ For an infinite lattice with periodic boundary conditions the semiconducting gap given by eq. (3) is $\Delta=0.83$ for $V=1$, and $\Delta=0.15$ for $V=0.4$.

${ }^{12}$ Our truncation error is reduced to $10^{-4}$ because we target the lowest two states. A targetted state is one whose energy and wavefunction we try to determine accurately.

13 The ground state would not be degenerate if the impurity were not in the middle of a lattice with open boundary conditions because this corresponds to an asymmetric double well potential.

${ }^{14}$ J. K. Furdyna, J. Appl. Phys. 64, R29 (1988).

15 J. B. Boyce and C. P. Slichter, Phys. Rev. B 13, 379 (1976). 


\section{FIGURES}

FIG. 1. c-spin-f-spin correlation functions for a symmetric Anderson impurity in a metal and a semiconducting host $(V=1)$ at half-filling. The correlations are between the f-spin of the impurity and the spins of the conduction electrons. $\mathrm{R}$ is the distance from the impurity site. ( $\left.L=25, t=1, V_{0}=1, U_{0}=8\right)$. Solid lines are guides to the eye. (a) Linear plot. Notice the Friedel oscillations in the metallic case. (b) Linear-log plot. In the semiconducting case the correlation functions die off very quickly due to the presence of the gap.

FIG. 2. c-spin-f-spin correlation functions for a symmetric Anderson impurity in a semiconducting host at half-filling. The correlations are between the f-spin of the impurity and the spins of the conduction electrons. $\mathrm{R}$ is the distance from the impurity site. $\left(L=25, N_{e l}=50\right.$, $t=1, V_{0}=1, U_{0}=8$ ). The correlation length increases as $V$ (and the gap) decreases. Solid lines are guides to the eye.

FIG. 3. A symmetric Anderson impurity in a half-filled semiconductor doped with one hole: (a) Hole density versus site, (b) Spin density versus site. For large $V_{0}$ both the hole and the spin are spread out over the lattice. For small $V_{0}$ they are localized near the impurity which is on site $i=0 . L=25, N_{e l}=49, t=1, V=1, U_{0}=8$. Solid lines are guides to the eye.

FIG. 4. Kinetic energy $\left(-t<c_{i \sigma}^{\dagger} c_{i+1 \sigma}+c_{i+1 \sigma}^{\dagger} c_{i \sigma}>\right)$ of a bond between site $i$ and site $i+1$ versus site $i$ for a hole in a semiconductor with a symmetric Anderson impurity. $L=25, N_{e l}=49$, $t=1, V=1, U_{0}=8$. Notice that the bonds connecting the impurity site have the largest magnitude of the kinetic energy for the smallest values of $V_{0}$. Solid lines are guides to the eye. 
FIG. 5. The z-component of the total spin in the f-orbitals and in the conduction orbitals for a symmetric Anderson impurity in a half-filled semiconductor (solid symbols, $V=1, N_{e l}=49$ ) doped with a hole versus $V_{0}$. The circles are for the total f-spin $\left(S_{z}^{f}=\sum_{i} S_{z}^{f}(i)\right)$ and the squares are for the total conduction $\operatorname{spin}\left(S_{z}^{c}=\sum_{i} S_{z}^{c}(i)\right) . \mathrm{L}=25, t=1$, and $U_{0}=8$. Solid lines are guides to the eye.

FIG. 6. A symmetric Anderson impurity in a half-filled metal doped with one hole: (a) Hole density versus site, (b) Spin density versus site. The hole is never localized at the impurity site $(i=0)$ but the spin density is localized for small $V_{0} . L=25, N_{e l}=25, t=1, U_{0}=8$. Solid lines are guides to the eye.

FIG. 7. Hole density versus site for a symmetric Anderson impurity in a half-filled semiconductor doped with two holes. $t=1, V=1, U_{0}=8, L=25, N_{e l}=48$, and $S_{z}=0$. For $V_{0}=0.1$ and 1.0, one hole is localized in the vicinity of the impurity and the other is spread out over the host lattice. For $V_{0}=0.1$ and 1.0, the ground state is nearly degenerate; the data shown are for the triplet state; the data for the singlet state are identical. For $V_{0}=2.0$ and 10.0, a singlet forms at the impurity site, and the two holes are spread over the rest of the lattice. For $V_{0}=2.0$ and 10.0, the ground state is a nondegenerate singlet.

FIG. 8. The chemical potential $\mu=E(N)-E(N-1)$ versus the electron filling $N$ for $t=1$, $U_{0}=8, V=1$, and $L=25$. The impurity is located in the middle of the symmetric Anderson lattice. The case of no impurity is shown for comparison. Solid lines are guides to the eye. 
Figure 1a

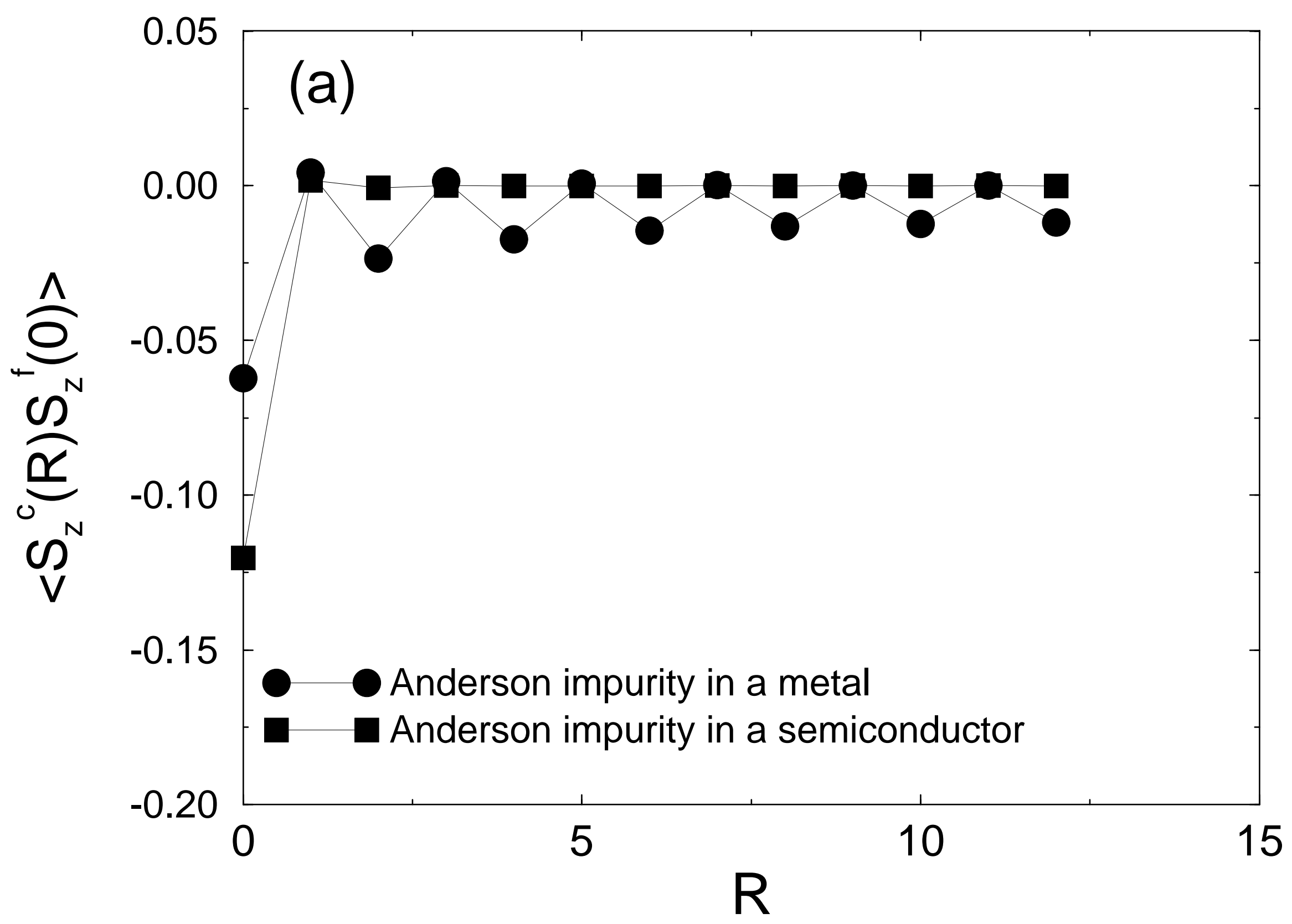


Figure 1b

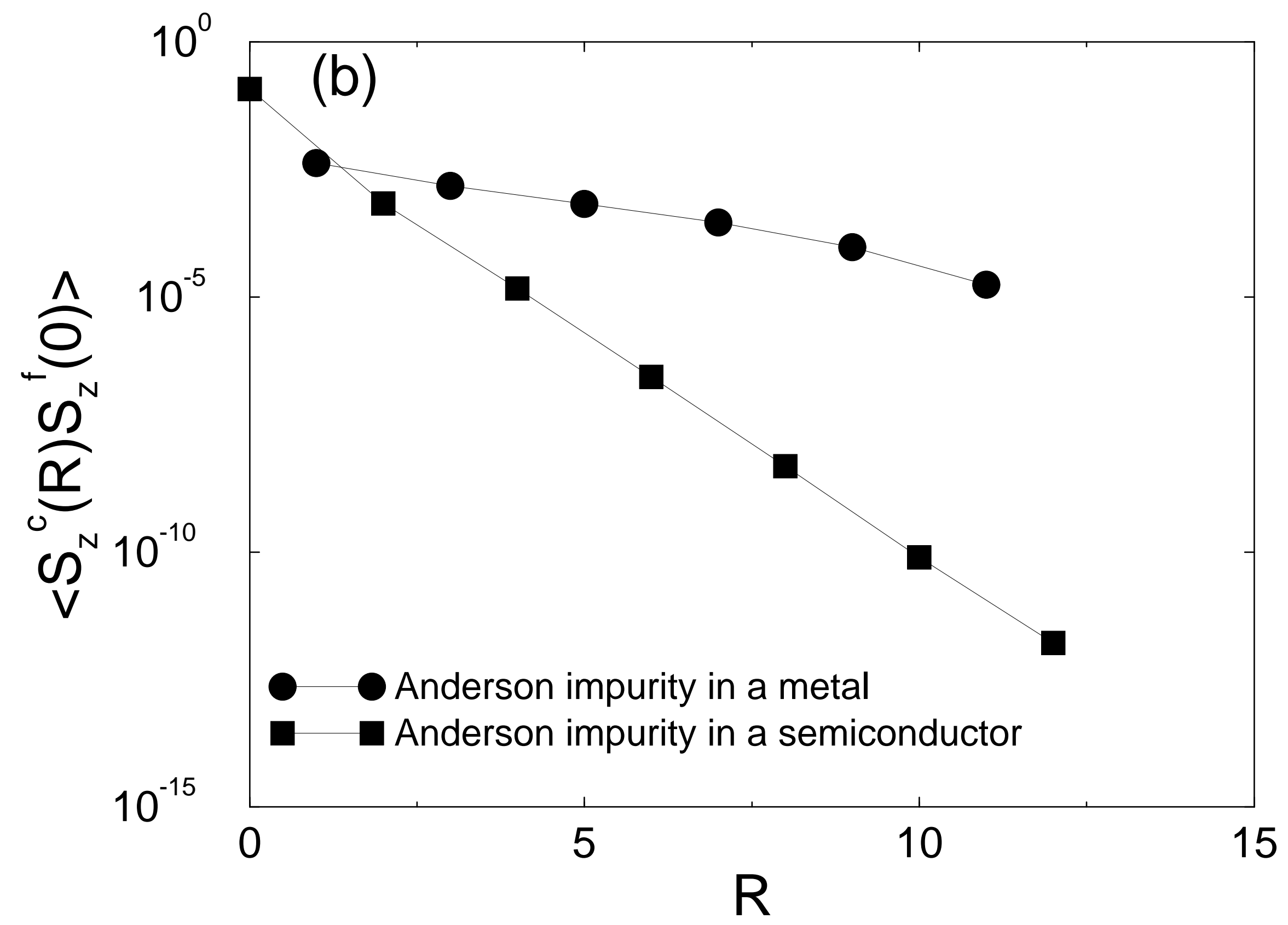


Figure 2

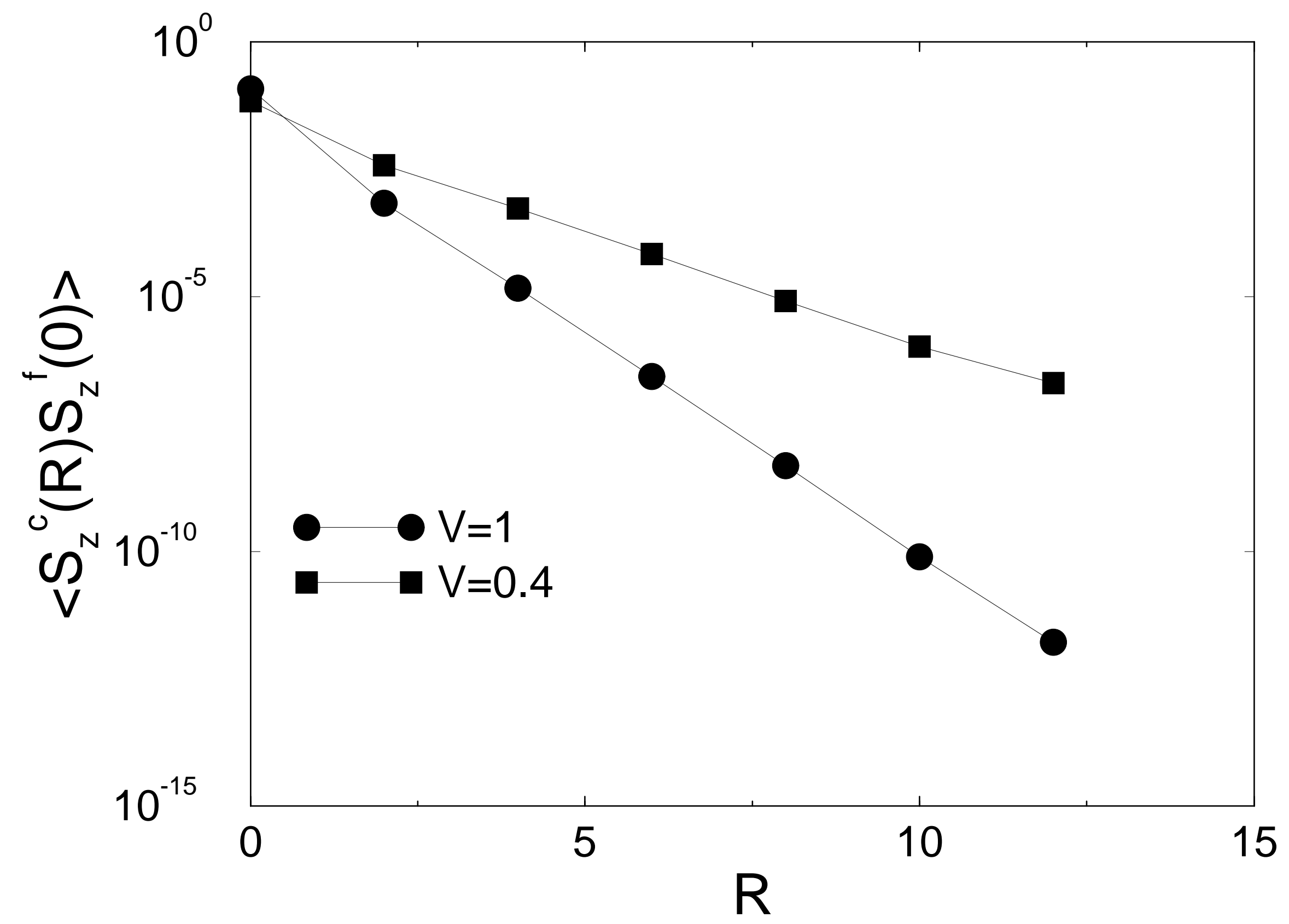


Figure $3 a$

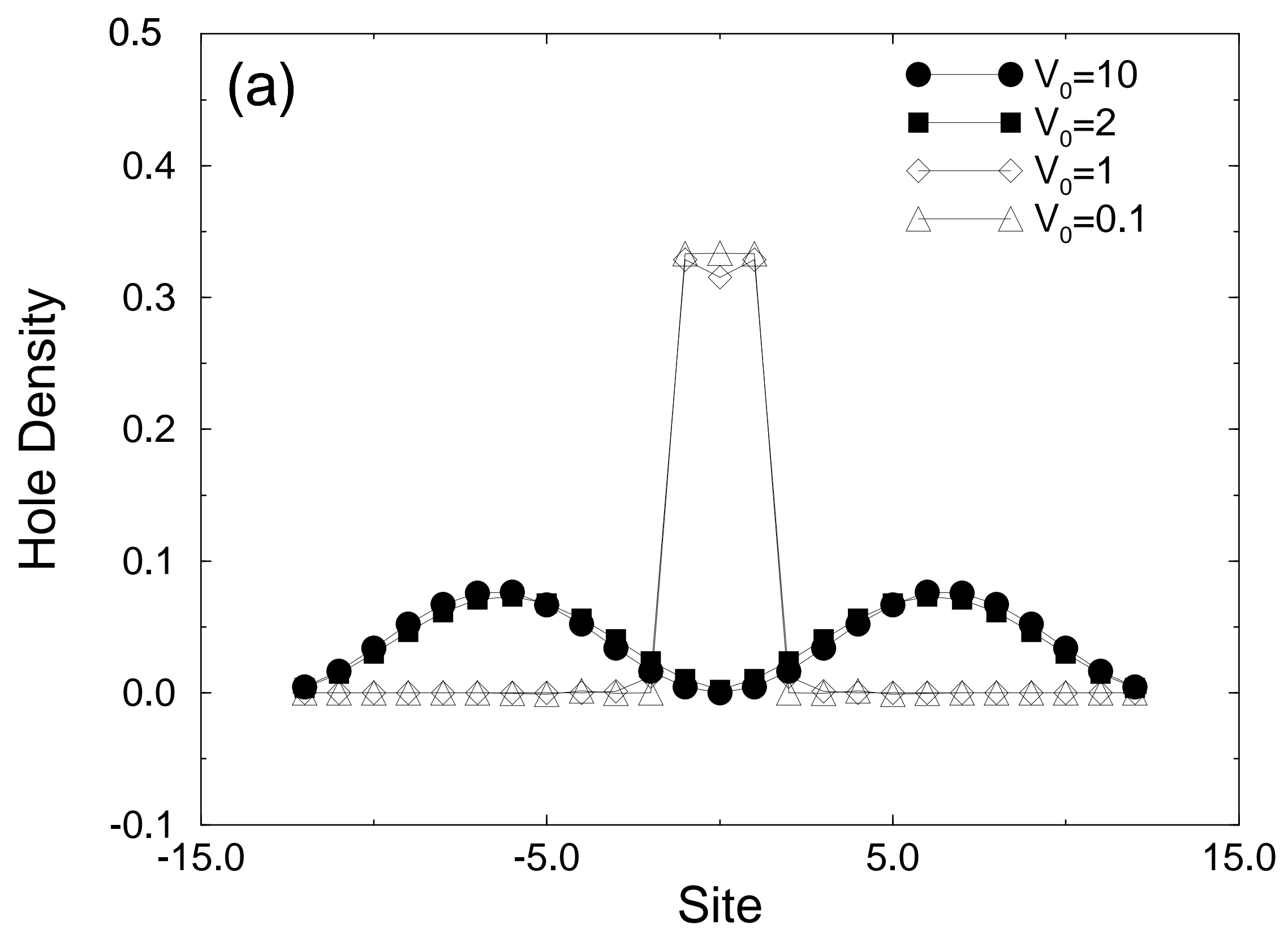


Figure $3 b$

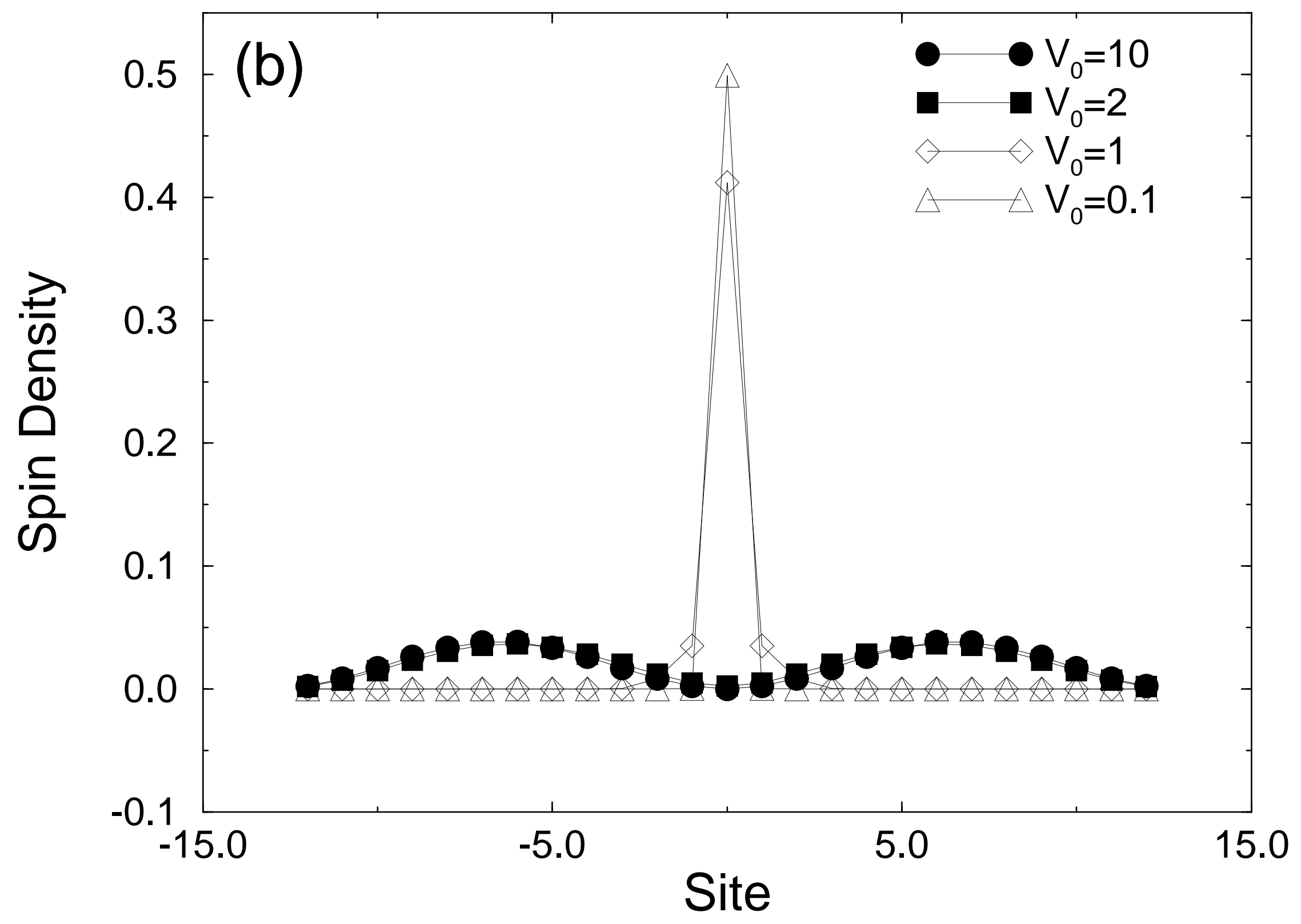


Figure 4

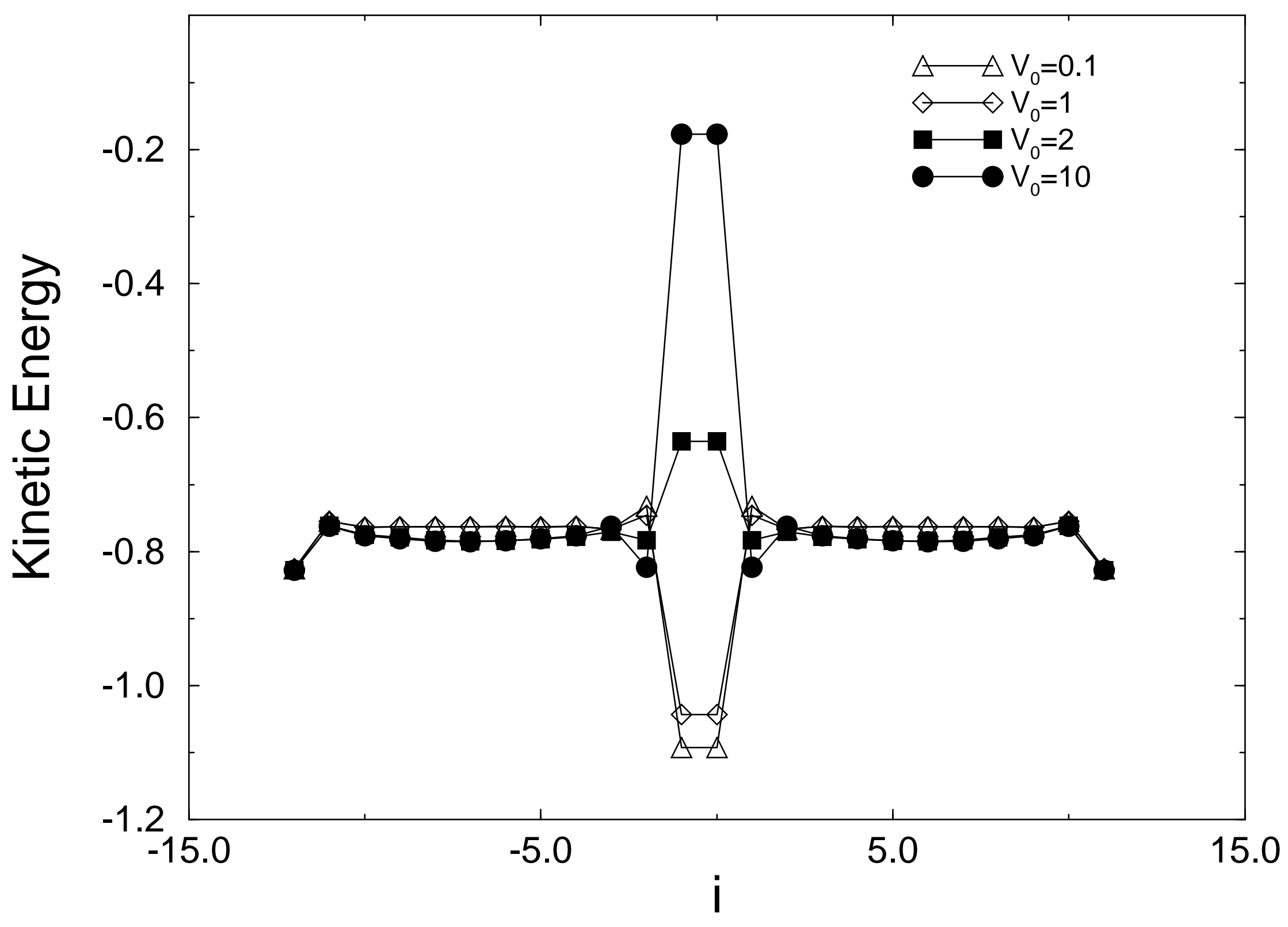


Figure 5

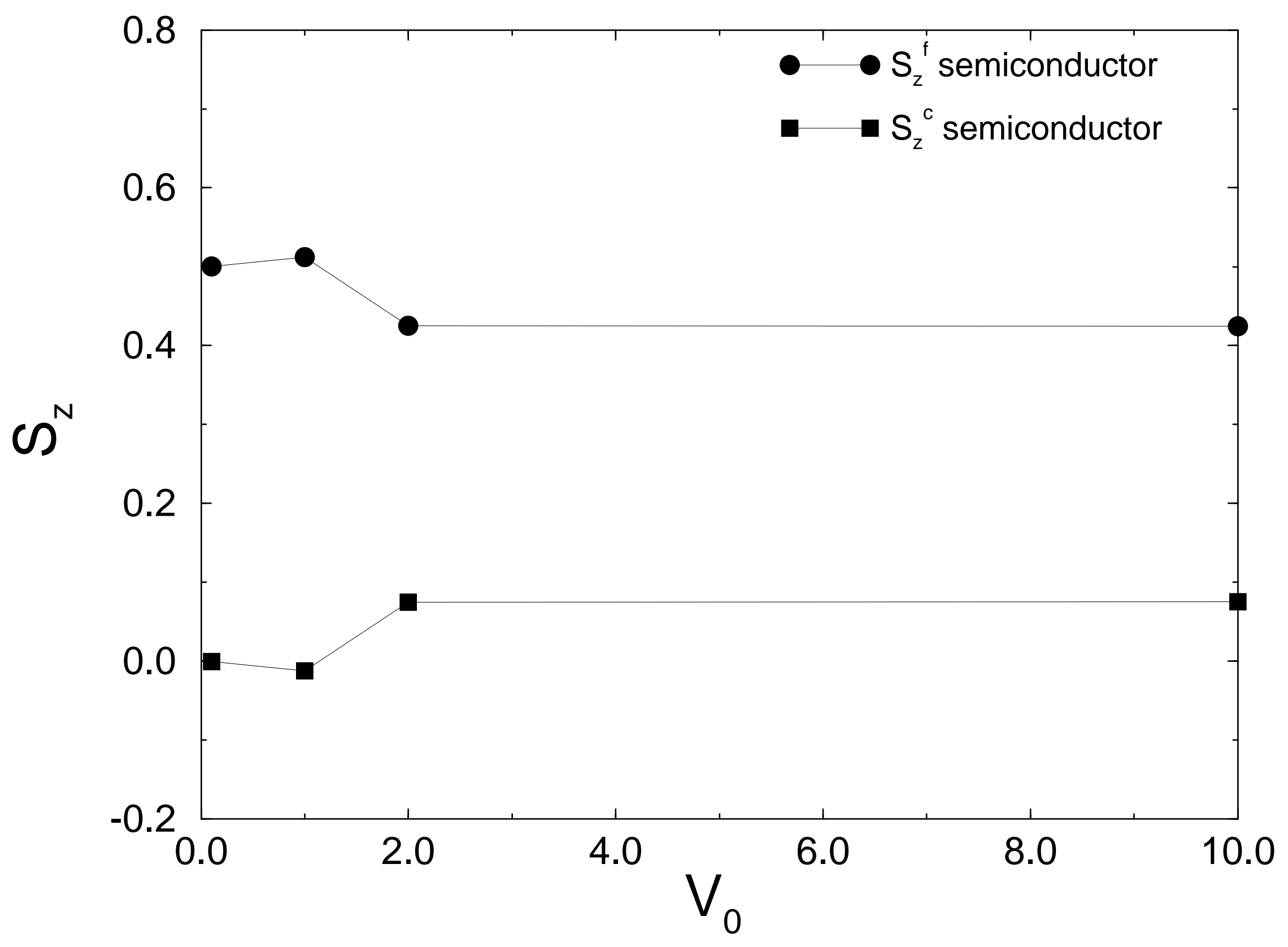


Figure $6 a$

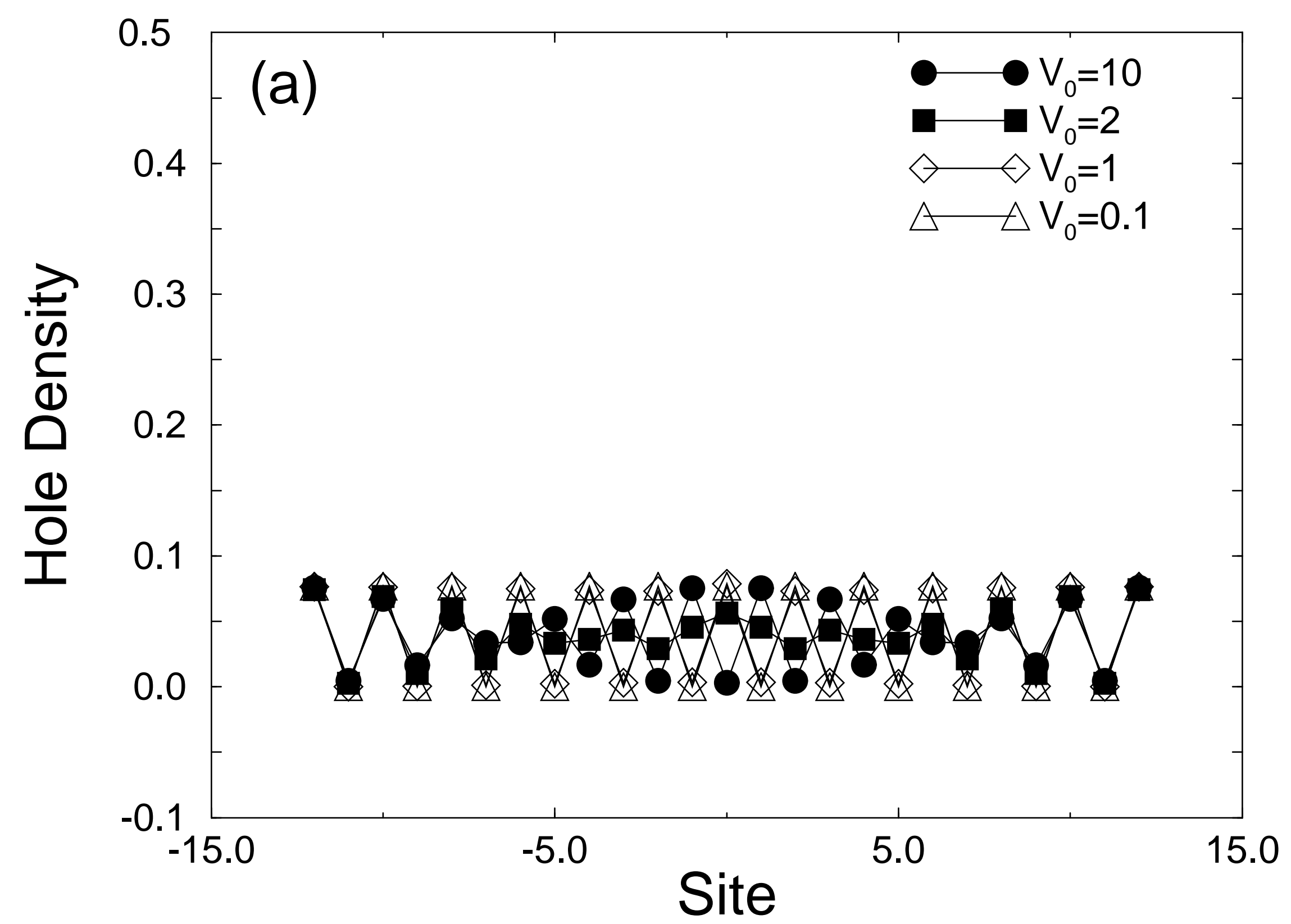


Figure $6 b$

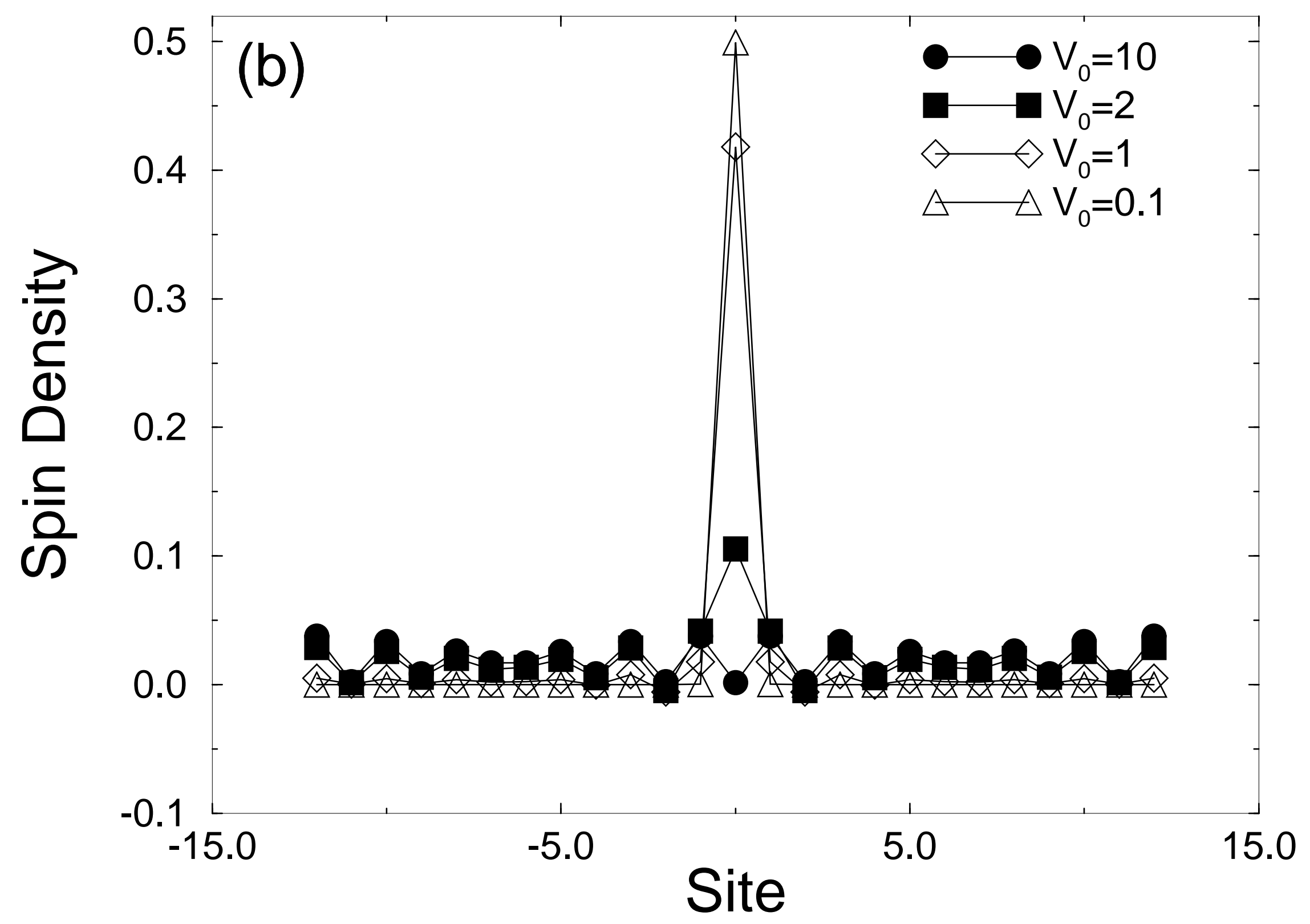


Figure 7

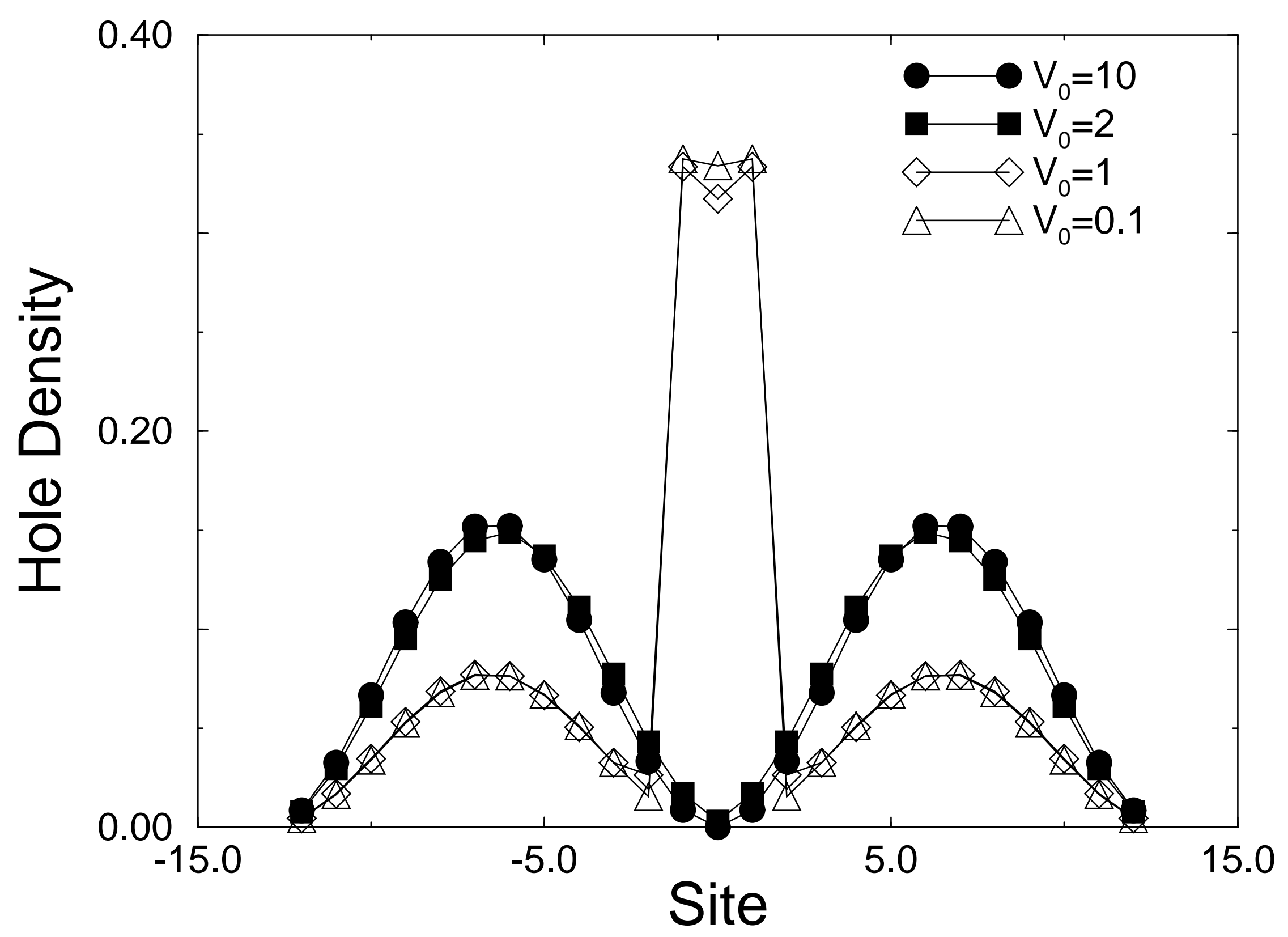


Figure 8

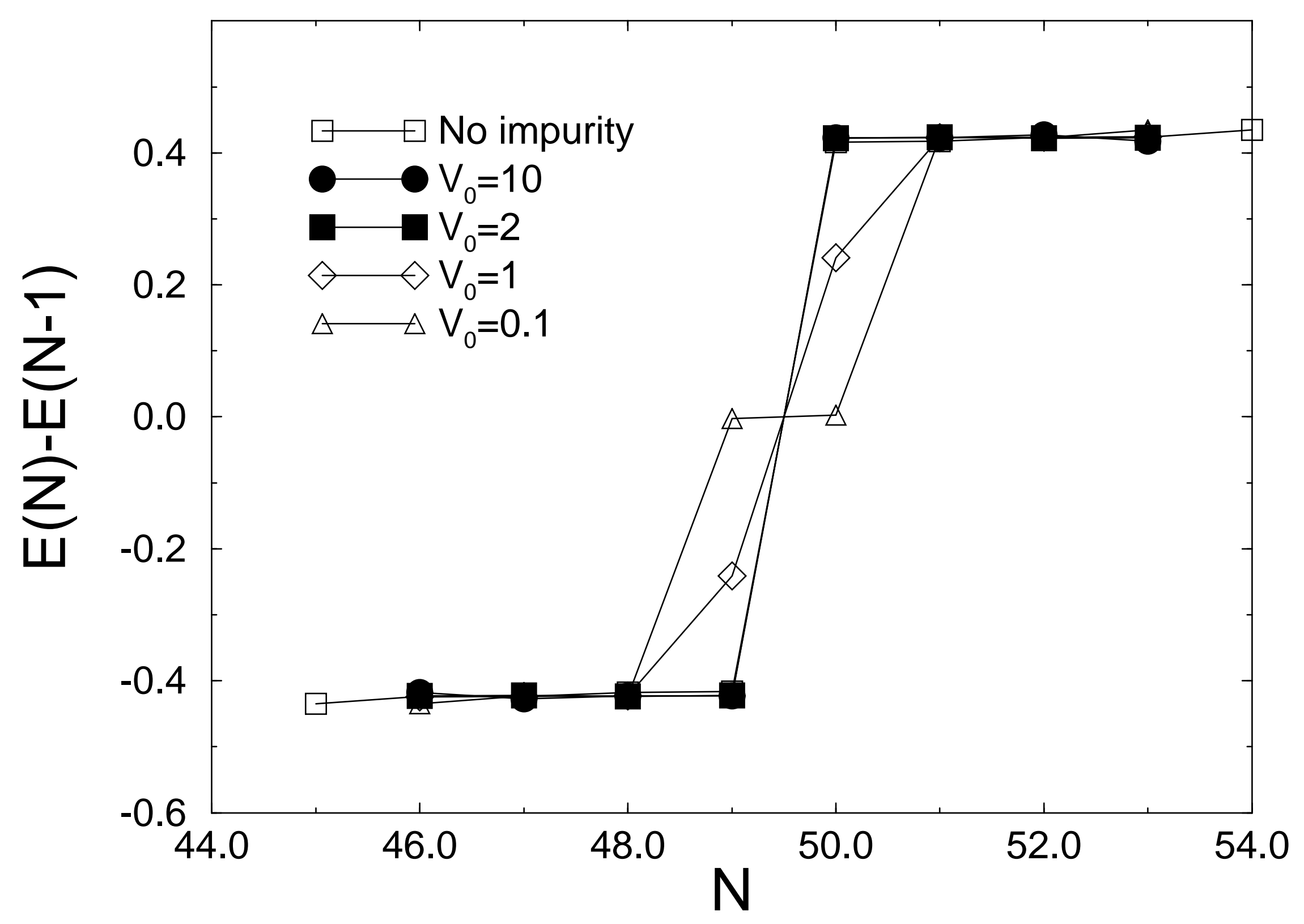

